\title{
Omomyc expression in skin prevents Myc-induced papillomatosis
}

\author{
L Soucek $^{\star 1,2}$, S Nasi ${ }^{2}$ and GI Evan ${ }^{1}$ \\ 1 Cancer Research Institute, UCSF, San Francisco, CA, USA \\ 2 Istituto di Biologia e Patologia Molecolari, CNR, Universita' La Sapienza, \\ Roma, Italy \\ * Corresponding author: L Soucek, Cancer Research Institute, UCSF, 2340 \\ Sutter Street, San Francisco, CA 94143-0875, USA. Tel: 415514 0760; \\ fax: 415 5140878; E-mail: Isoucek@cc.ucsf.edu
}

Received 12.5.03; revised 14.10.03; accepted 18.2.04; published online 14.5.04 Edited by DR Green

\begin{abstract}
Obligate sensitization to apoptosis provides a safeguard mechanism against the oncogenic potential of Myc. Omomyc is a mutant bHLHZip domain that sequesters Myc in complexes that are unable to bind to the $\mathrm{E}$ box recognition element and activate transcription but remain competent for transcriptional repression. Omomyc has the peculiar properties of reverting Myc-induced transformation of tissue culture cells and enhancing Myc proapoptotic function. Thus, Omomyc has the potential to act as a potent suppressor of Myc-induced oncogenesis. To validate the therapeutic potential of Omomyc in vivo, we targeted its expression to the adult suprabasal epidermis of $I n v-c-M y c E R^{T A M}$ transgenic mice which express a switchable form of the Myc protein in suprabasal cells. Activation of Myc induces rapid epidermal hyperplasia and papillomatosis. We show that Omomyc inhibits such Myc-induced papillomatosis, potentiating Mycdependent apoptosis in a tissue in which it is usually strongly suppressed. Furthermore, Omomyc expression restores the normal keratinocyte differentiation program and skin architecture, both of which are otherwise disrupted by Myc activation. These findings indicate that it is possible to selectively enhance the intrinsic apoptotic pathway mediated by Myc and so quell its oncogenic action.

Cell Death and Differentiation (2004) 11, 1038-1045.

doi:10.1038/sj.cdd. 4401443

Published online 14 May 2004
\end{abstract}

Keywords: Omomyc; Myc; apoptosis; p53; Bcl- $\mathrm{x}_{\mathrm{L}}$

Abbreviations: $4 \mathrm{OHT}$, 4-hydroxytamoxifen; Inv, involucrin

\section{Introduction}

The proto-oncogene $c-m y c$ encodes the Myc basic helixloop-helix leucine zipper (bHLHZip) transcription factor which, upon dimerization with its partner Max, binds the E-box DNA sequence CACGTG. ${ }^{1}$ Deregulated and elevated myc expression, arising through translocation, amplification, mutation or sustained signaling by upstream activating pathways is widespread in cancer cells of many types, including lymphoma, neuroblastoma, small cell lung carcinoma, breast and colon cancer. ${ }^{2}$ In normal cells, Myc plays a pivotal role in mediating $\mathrm{G} 1$ progression and the $\mathrm{G} 1-\mathrm{S}$ phase transition and in its absence cells are impaired in proliferation. ${ }^{3}$ Consistent with this, c-myc haploinsufficiency results in reduced body size while targeted ablation of the c-myc gene in mice causes embryonic lethality with failure of multiple proliferating cell lineages. ${ }^{4}$ Paradoxically, however, Myc can also induce cell death, 5,6 most markedly in the absence of survival factors or in combination with various cellular stresses.

Omomyc is a c-Myc-derived mutant bHLHZip domain generated by substituting four amino acids within the c-Myc leucine zipper that confer altered dimerization specificity and allow Omomyc to form homodimers and heterodimerize with wild-type Myc. We have recently shown that Omomyc potentiates Myc-dependent apoptosis in a manner dependent on Myc expression levels. Overexpressed Omomyc competes with Max for Myc and thereby prevents Myc from binding to the E-box and activating transcription. ${ }^{7}$ In contrast, Myc-dependent trans-repression, which does not involve binding to the E-box, is unaffected, or even enhanced, by Omomyc. ${ }^{8}$ The strong proapoptotic activity of Omomyc mutant protein may spring from its ability to differentially influence activated and repressed Myc transcriptional targets.

To determine how Omomyc potentiates apoptosis, we have investigated its effects on known effector pathways thought to be important in determining Myc-dependent apoptosis, in particular the ARF-p53 pathway and the mitochondrial program regulated by $\mathrm{Bcl}-2 / \mathrm{Bax}$ proteins that leads to holocytochrome $c$ release..$^{9,10}$ We show that Omomyc can override some, but not all, factors that inhibit Myc-induced apoptosis.

Since Omomyc inhibits Myc-induced proliferation while potentiating Myc-dependent apoptosis, it has the intriguing potential to suppress or reverse Myc-induced tumorigenesis. We decided to test this using our previously described model of skin tumorigenesis in which activation of the 4-hydroxytamoxifen (4-OHT)-dependent switchable form of Myc (MycER ${ }^{\text {TAM }}$ ) in suprabasal epidermis induces the rapid onset of keratinocyte proliferation. ${ }^{11}$ Myc-induced apoptosis is innately suppressed in intact skin, so activation of Myc rapidly leads to profound hyperplasia and dramatic papilloma formation. We therefore chose this model to assess the ability of Omomyc in vivo to potentiate Myc-induced apoptosis and antagonize Myc-induced papillomatosis.

\section{Results}

\section{Omomyc overcomes inhibition of Myc-induced apoptosis by $\mathrm{Bcl}-\mathrm{x}_{\mathrm{L}}$}

Overexpression of $\mathrm{Bcl}-2$ or $\mathrm{Bcl}-\mathrm{x}_{\mathrm{L}}$ blocks Myc-induced release of mitochondrial holocytochrome $c$ and consequent apoptosis..$^{9,12}$ To determine the susceptibility of Omomycpotentiated apoptosis to suppression by $\mathrm{Bcl}-2 / \mathrm{Bcl}-\mathrm{x}_{\mathrm{L}}, \mathrm{Bcl}-\mathrm{x}_{\mathrm{L}}$ 
overexpressing Rat-1 cells were infected with the MycER ${ }^{\text {TAM }}$ retroviruses and selected with puromycin. Then, a second round of transient infection was performed with a retrovirus directing expression of Omomyc together with the fluorescent marker protein GFP driven from an IRES. ${ }^{13}$ Immediately after this second infection, cells were serum-deprived for $24 \mathrm{~h}$, in the presence of either 4-OHT (to activate MycER ${ }^{\text {TAM}}$ ) or control EtOH carrier. Flow cytometry was used to evaluate the extent of apoptosis in GFP + cells. Rat-1MycER ${ }^{\text {TAM }}$ cells showed $30 \%$ apoptosis in low serum even in the absence of 4-OHT, similar to wild-type Rat-1 cells (data not shown). Myc activation caused a significant increase in apoptosis which was strongly enhanced by Omomyc, as expected. ${ }^{8}$ Although $\mathrm{Bcl}-\mathrm{x}_{\mathrm{L}}$ overexpression significantly impaired Mycinduced apoptosis, it had negligible effect on apoptosis induced by coexpression of both Myc and Omomyc (Figure 1).

\section{Omomyc-induced apoptosis is p53 dependent but ARF independent}

The ARF-Mdm2-p53 pathway is considered important for the efficient induction of apoptosis in many cell types, including fibroblasts. ${ }^{14}$ To determine whether ARF and p53 are obligate mediators of Omomyc-dependent proapoptotic activity, wild type, $\mathrm{ARF}^{-1-}$, and $\mathrm{p} 53^{-/-} \mathrm{MEFs}$ were infected with retrovirus directing expression of MycER ${ }^{T A M}$ and then transiently infected with Omomyc/IRES-GFP retrovirus. After $24 \mathrm{~h}$ of

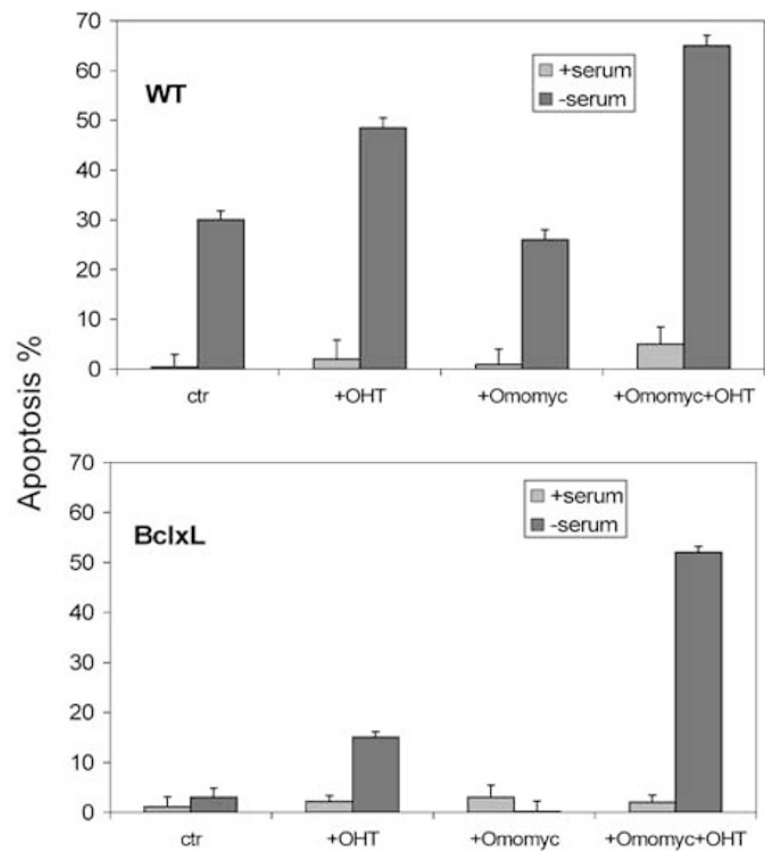

Figure 1 Omomyc overcomes $\mathrm{Bcl}-\mathrm{x}_{\mathrm{L}}$-mediated suppression of apoptosis. Control (top panel) or Bcl- $\mathrm{x}_{\mathrm{L}}$ overexpressing (bottom panel) Rat-1/MycER ${ }^{\text {TAM }}$ fibroblasts were treated with either EtOH or $4-\mathrm{OHT}$ for $24 \mathrm{~h}$ and then serumdeprived for a further $24 \mathrm{~h}$. The percentage of apoptotic nuclei in the culture was then determined by Annexin V staining and flow cytometric analysis. Ctr, cells treated with $\mathrm{EtOH} ;+\mathrm{OHT}$, cells treated with 4-hydroxytamoxifen; Omomyc cells treated with $\mathrm{EtOH}$ and infected with the Omomyc retroviral vector; + $\mathrm{OHT}$ + Omomyc: cells treated with 4-hydroxytamoxifen and infected with the Omomyc retroviral vector serum deprivation, either with or without 4-OHT, apoptosis was assessed by flow cytometry. Apoptosis induced by Myc alone was significantly reduced in the absence of either ARF or p53. In contrast, although Omomyc/Myc-induced apoptosis was also impaired by the absence of p53, it was not inhibited in ARF-deficient cells (Figure 2). Thus, potentiation of Mycinduced apoptosis by Omomyc is dependent on p53 but independent of ARF.

\section{Generation and characterization of Inv-Omomyc transgenic mice}

We next asked whether Omomyc is able to potentiate Mycinduced apoptosis in vivo. We chose skin epidermis since this is a tissue in which Myc efficiently drives cell proliferation but where Myc-induced apoptosis is innately suppressed. Consequently, Myc activation is sufficient to induce profound hyperplasia and tumorigenesis. ${ }^{11}$
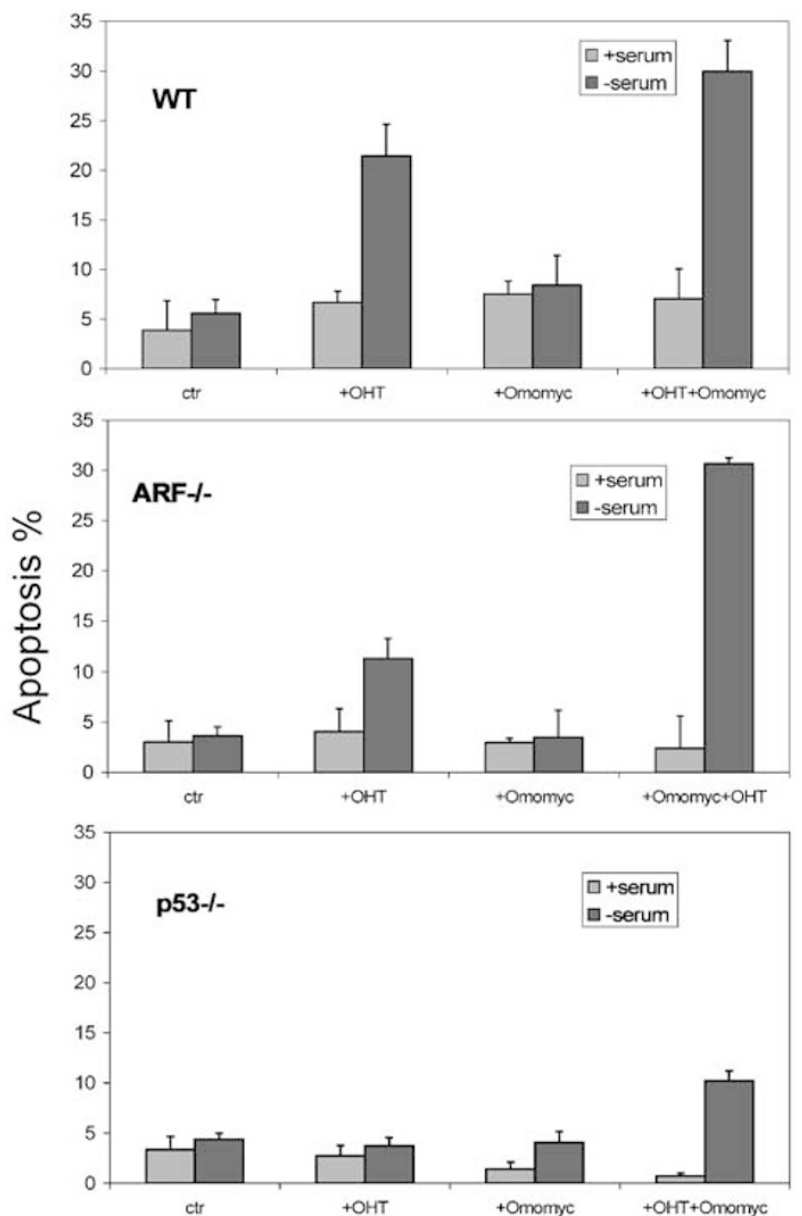

Figure 2 Omomyc-induced apoptosis is ARF independent and p53 dependent. Wild type (top panel), $\mathrm{ARF}^{-1-}$ (middle panel) or $\mathrm{p5}^{-1-}$ (bottom panel) MEFs expressing MycER ${ }^{\text {TAM }}$, treated with either EtOH or 4-OHT, were serum-deprived for $24 \mathrm{~h}$ and apoptotic nuclei then scored by Annexin V staining and flow cytometric analysis. Comparison of the top and middle panels shows that absence of ARF greatly reduces Myc-dependent apoptosis, but not apoptosis induced by Omomyc and Myc together. In contrast, absence of p53 significantly impairs both Myc- and Myc/Omomyc-induced apoptosis 
Expression of Omomyc was targeted to suprabasal keratinocytes using the human involucrin promoter (Figure $3 \mathrm{~A})^{11,15}$ and expression of Omomyc protein in skin in vivo assessed immunohistochemically using a rabbit antiOmomyc antibody raised against the leucine zipper mutated region. This antibody exhibited no significant binding to epidermis of single transgenic inv-c-MycE $R^{T A M}$ mice, indicating lack of cross-reaction with Myc-ER ${ }^{\text {TAM }}$. In contrast, antibody reactivity in inv-Omomyc transgenic epidermis was evident in the great majority of suprabasal interfollicular keratinocytes and in the inner root sheath (IRS) of hair follicles (Figure 3B), correlating well with the established distribution of involucrin staining (data not shown). Binding by the antiOmomyc antibody was efficiently blocked by preincubation with the original Omomyc peptide immunogen, confirming the antigen specificity of the antibody. Despite widespread suprabasal expression of Omomyc, however, the skin of Inv-Omomyc transgenic mice appeared completely normal, indicating that Omomyc exerts no ostensible effect on its own. This is consistent with our previously published data showing that Omomyc displays biological activity only in presence of high levels of Myc. ${ }^{8}$

\section{Omomyc expression suppresses c-Myc-induced papillomatosis}

To explore the effects of Omomyc expression on Myc oncogenesis, Inv-Omomyc transgenic mice were outbred to establish independent founders. Two separate lines, each with similar Omomyc expression level, were identified and crossed with involucrin-c-MycER ${ }^{T A M}$ expressing mice ${ }^{11}$ to generate inv-Omomyc $\mathrm{X}$ inv-c-MycER ${ }^{T A M}$ double transgenic animals. Subsequent experiments were each conducted in triplicate, using three individual mice of each genotype and for each condition. We observed insignificant variation between individuals in each group.
Neither control single transgenic inv-c-MycE $R^{\text {TAM }}$ mice treated with ethanol carrier nor inv-Omomyc single transgenic mice treated with either $4 \mathrm{OHT}$ of ethanol carrier exhibited any evident phenotype (Figure 4A). However, as observed previously, sustained activation of suprabasal c-MycER ${ }^{\text {TAM }}$ by daily topical application of $4 \mathrm{OHT}$ to shaved dorsal skin of inv-c-MycE $R^{T A M}$ single transgenic mice for 21 days induced progressive epidermal hyperplasia and, in many cases, dysplasia. Histological analysis showed significant skin thickening with flaking cornified epidermis, scabby dry lesions and papillomatosis associated with hyperkeratosis and hypergranulosis, especially in the perifollicular area. In striking contrast, the skin of inv-c-MycER ${ }^{T A M} /$ Omomyc double transgenic mice treated for 21 days with $4-\mathrm{OHT}$ remained perfectly normal (Figure 4B).

To exclude the trivial possibility that Omomyc somehow suppresses c-MycER ${ }^{\text {TAM }}$ expression, dorsal skin sections from inv-c-MycER ${ }^{T A M} / O m o m y c$ double transgenic mice were stained with anti-ER ${ }^{\text {TAM }}$ antibody. This showed that the double transgenic mice clearly overexpress $\mathrm{C}^{-M y c E R^{T A M}}$ in their suprabasal epidermis (Figure 5). Thus, Omomyc suppresses c-MycER ${ }^{\text {TAM }}$ induced keratinocyte hyperplasia and papillomatous despite sustained expression of $\mathrm{C}-\mathrm{MycER}^{\mathrm{TAM}}$.

\section{Omomyc expression restores normal suprabasal keratinocyte differentiation that is otherwise disrupted by c-Myc activation}

Expression of Myc inhibits the normal differentiation program of suprabasal keratinocytes, leading to retention of early suprabasal markers, failure of the terminal enucleation characteristic of mature squames and accumulation of lessdifferentiated granular keratinocytes. To address whether Omomyc interferes with this Myc-dependent inhibition of keratinocyte differentiation, we used immunohistochemistry

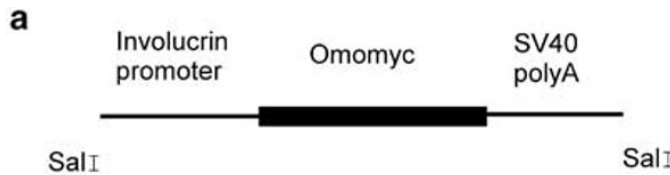

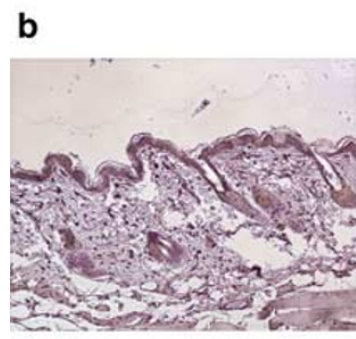

Omomyc

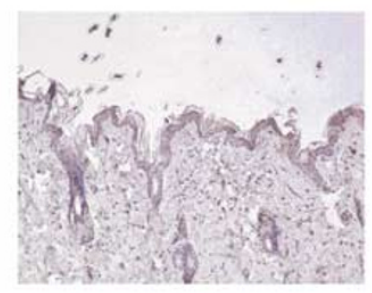

Omomyc + peptide

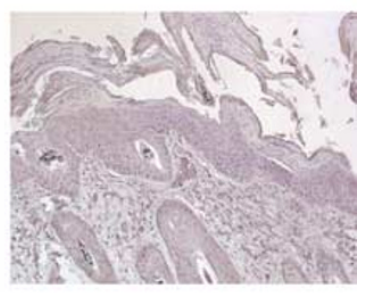

$\mathrm{MycER}+\mathrm{OHT}$

Figure 3 Generation of inv-Omomyc transgenic mice. (a)Transgene construction. The DNA encoding Omomyc was cloned downstream of the Involucrin promoter. (b) Transgene protein expression. Paraffin-embedded skin sections from Inv-Omomyc and Inv-c-MycER ${ }^{T A M}$ transgenic mice were stained with Omomyc antibody. Omomyc expression is present in the Inv-Omomyc epidermis (first panel). Omomyc staining is specifically blocked by preincubation of the antibody with its immunogenic peptide (second panel). No Omomyc staining is detectable in stained sections of Inv-c-MycER ${ }^{\text {TAM }}$ skin (third panel) 
A

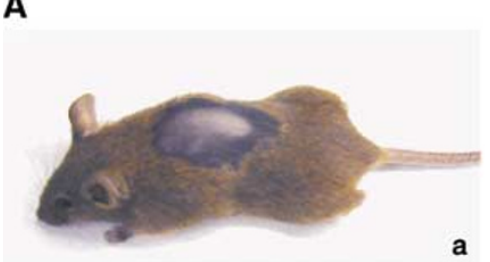

B
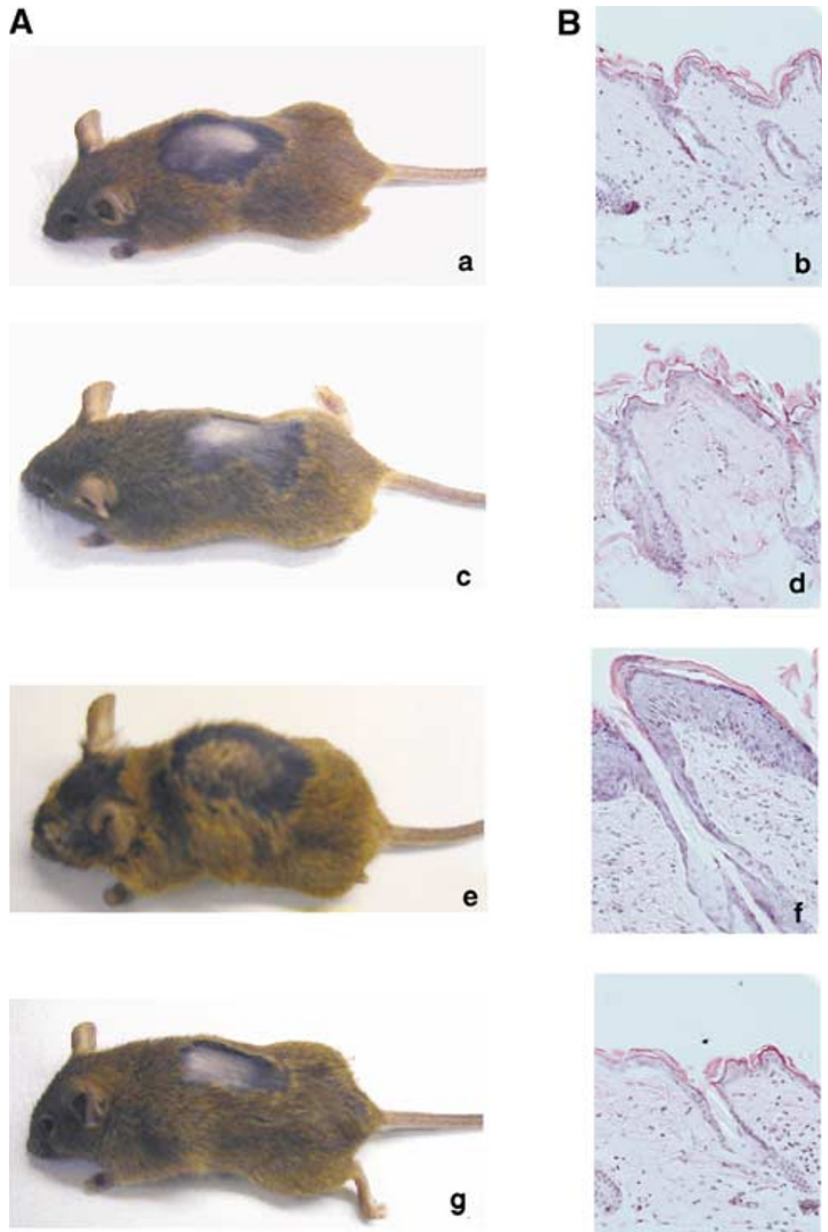

Figure 4 Omomyc expression suppresses Myc-induced papillomatosis in vivo. (A) Three each of Inv-c-MycER ${ }^{\text {TAM }}$, Inv-Omomyc single transgenic, and Inv-cMycER $R^{\text {TAM} / O m o m y c ~ d o u b l e ~ t r a n s g e n i c ~ m i c e ~ w e r e ~ t r e a t e d ~ w i t h ~ e i t h e r ~ e t h a n o l ~ o r ~}$ 4-OHT daily for 21 days, as described in Materials and Methods. Ethanol-treated Inv-c-MycER ${ }^{\text {TAM }}$ mice (a) and 4-OHT-treated Inv-Omomyc single transgenics (b) display normal skin, while Inv-c-MycER TAM transgenic mice treated with 4-OHT exhibit papillomas and appear scabby and sick (c). 4-OHT-treated Inv-c$M y c E R^{T A M} / O m o m y c$ double transgenic mice are indistinguishable from control mice (d). (B) $H$ \& E staining of dorsal skin sections from mice. Tissue sections from ethanol-treated control Inv-c-MycER ${ }^{\text {TAM }}$ mice (e) or Inv-Omomyc single transgenic animals (f) display normal skin architecture, whereas sections from inv-MycER $R^{T A M}$ transgenic mice treated with 4-OHT show thickened skin with several suprabasal layers (g). Tissue sections from Inv-c-MycER $R^{T A M} / O m o m y c$ double transgenics resemble those derived from control mice (h). The Figure shows representative sections of skin

to localize expression of two major differentiation-specific keratins, the suprabasal marker Keratin 1 (K1) and Keratin 14 (K14) which is normally restricted to basal keratinocytes. ${ }^{16,17}$

$\mathrm{K} 1$ staining was generally weak in the hyperplastic suprabasal areas induced by sustained activation of MycER ${ }^{\text {TAM }}$ for 3 weeks in inv-c-MycER ${ }^{\text {TAM }}$ single transgenic mice. In contrast, $\mathrm{K} 1$ expression remained high in the spinous and granular suprabasal cells of 3 week-4-OHT-treated skin of inv-MycER ${ }^{\text {TAM }} /$ Omomyc double transgenic mice, as it did in control mice and in inv-Omomyc single transgenic animals (Figure 6A). After 3 weeks of sustained c-MycER ${ }^{\text {TAM }}$ activation in inv-c-MycER ${ }^{\text {TAM }}$ single transgenic mice, K14

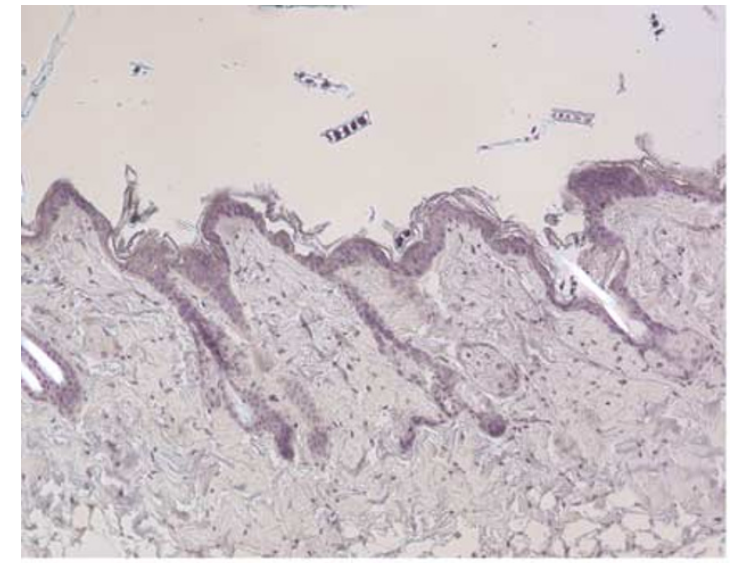

Omomyc + OHT

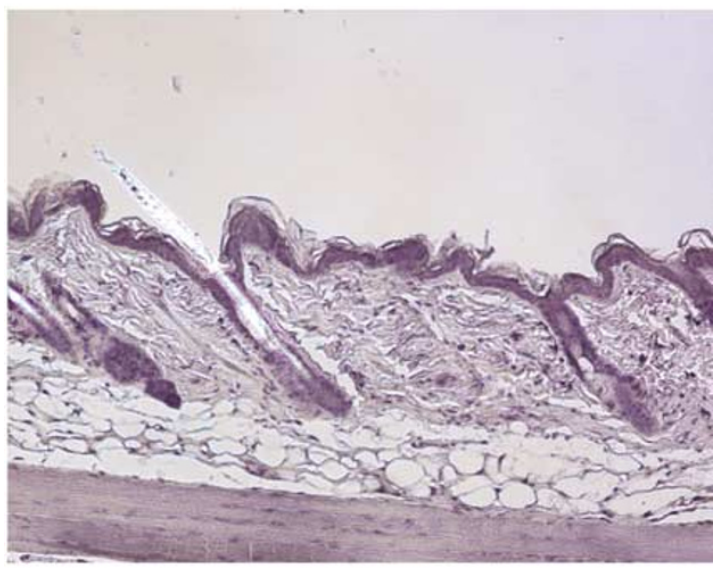

MycER ${ }^{\text {TAM } / O m o m y c ~+~ O H T ~}$

Figure $5 \mathrm{MycER}^{\text {TAM }}$ protein expression in Inv-MycER ${ }^{\text {TAM }} /$ Omomyc double transgenic mice. Tissue sections from Inv-Omomyc transgenic mice (top panel) and Inv-c-MycER $R^{\text {TAM} / O m o m y c ~ d o u b l e ~ t r a n s g e n i c ~ m i c e ~(b o t t o m ~ p a n e l) ~ w e r e ~}$ stained with estrogen receptor antibody to identify MycER ${ }^{\text {TAM}}$. MycER ${ }^{\text {TAM }}$ expression is present in the double transgenic mice but not in Omomyc single transgenics

expression extends well into the hyperplastic suprabasal compartment. In contrast, inv-c-MycER ${ }^{T A M} /$ Omomyc double transgenics, treated for 21 days with 4-OHT, exhibited the same basally restricted distribution of K14 staining as did control mice or inv-Omomyc single transgenic animals (Figure 6B). Thus, expression of Omomyc preserves the normal epidermal keratinocyte differentiation program despite the sustained presence of activated Myc.

\section{Omomyc promotes apoptosis in Myc overexpressing epidermis in vivo and in vitro}

Expression of Omomyc potentiates c-Myc-induced apoptosis in tissue culture cells. ${ }^{8}$ We used TUNEL staining to determine whether the ability of Omomyc to suppress Myc-induced skin papillomatosis might be due to potentiation of Myc-induced apoptosis in intact skin epidermis in vivo.

Very little apoptosis is evident in normal interfollicular skin or in skin from untreated inv-MycER TAM mice. Likewise, we 
A
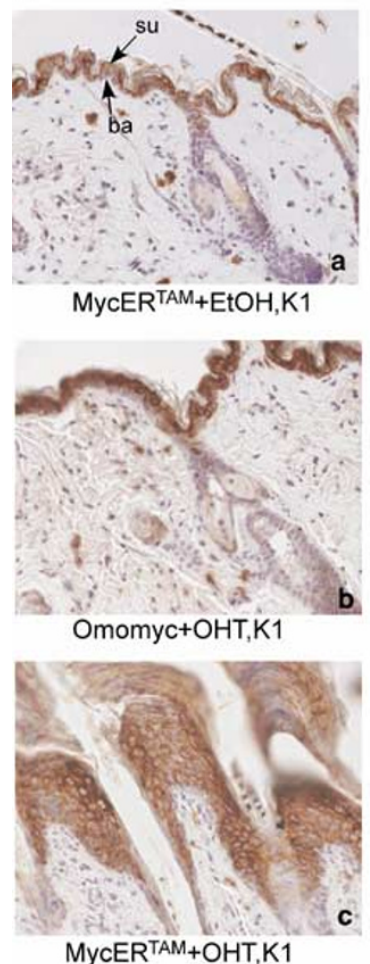

$\mathrm{MycER}^{\mathrm{TAM}}+\mathrm{OHT}, \mathrm{K}$

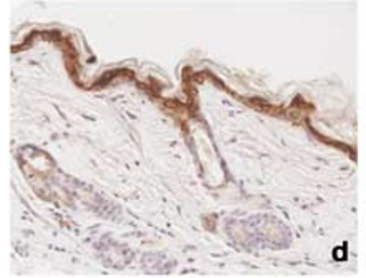

MycERTAM/Omomyc+OHT,K1
B

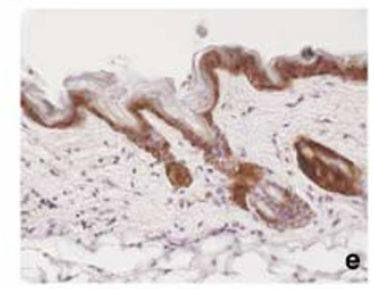

$M y c R^{T A M}+E t O H, K 14$

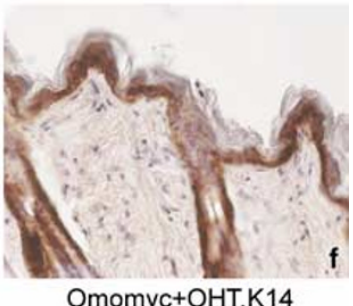

Omomyc+OHT,K14

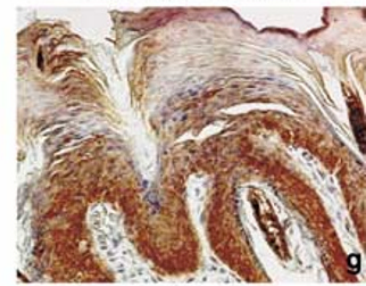

MycER ${ }^{T A M}+\mathrm{OHT}, \mathrm{K} 14$

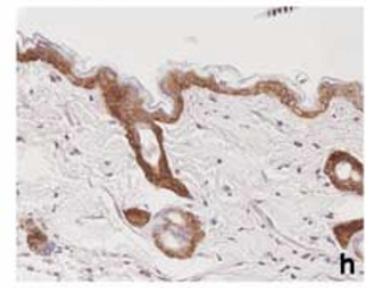

MycER TAM/Omomyc+OHT,K14

Figure 6 Omomyc restores normal keratinocyte differentiation. (A) Dorsal skin sections from inv-MycER ${ }^{T A M}$ and inv-Omomyc single transgenic, and from Inv-cMycER ${ }^{\text {TAM }} /$ Omomyc double transgenic mice all stained for the suprabasal marker Keratin 1. In papillomas from Inv-c-MycER ${ }^{T A M}$ mice treated with 4-OHT for 21 days, Keratin 1 staining is weak but distributed throughout the hyperplastic suprabasal layer (c). In contrast, the distribution of Keratin 1 in Inv-c-MycER ${ }^{\text {TAM }}$, Omomyc double transgenic skin (d) is identical to that in control skin and in skin of ethanol-treated control Inv-c-MycER ${ }^{T A M}$ transgenic mice (a) and inv-Omomyc single transgenics (b). (B) Skin sections from the same mice as in (a) stained for the basal marker Keratin 14. In papillomatous skin from Inv-c-MycER ${ }^{T A M}$ mice treated with $4 \mathrm{OHT}$ for 21 days, Keratin 14 staining extends well into the hyperplastic suprabasal layers (g). In ethanol-treated control Inv-c-MycER ${ }^{\text {TAM }}$ transgenic mice (e) and Inv-Omomyc single transgenics (f), Keratin 14 staining is confined to the basal layer, as it is in control skin. In inv-MycER $R^{\text {TAM } / O m o m y c}$ double transgenics Keratin 14 distribution resembles that of normal skin (h). su, suprabasal layer; ba, basal layer

saw no evidence of apoptosis in suprabasal skin of 4-OHTtreated inv-MycER ${ }^{\text {TAM }}$ mice, as previously reported, ${ }^{11}$ most probably because of the presence of abundant endogenous survival signals. ${ }^{11}$ However, widespread apoptosis was evident in the suprabasal layer of 4-OHT-treated inv-c$M y c E R^{T A M} /$ Omomyc double transgenic mice (Figure $7 \mathrm{~A}$ ). Thus, the combination of active Myc and Omomyc is a profound promoter of apoptosis in skin epidermis.

A

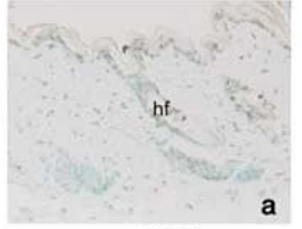

MycERTAM+EtOH

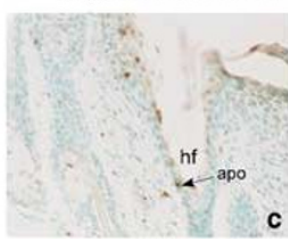

MycERTAM+OHT
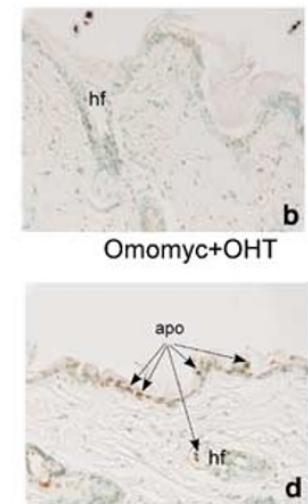

MycERTAM/Omomyc+OHT

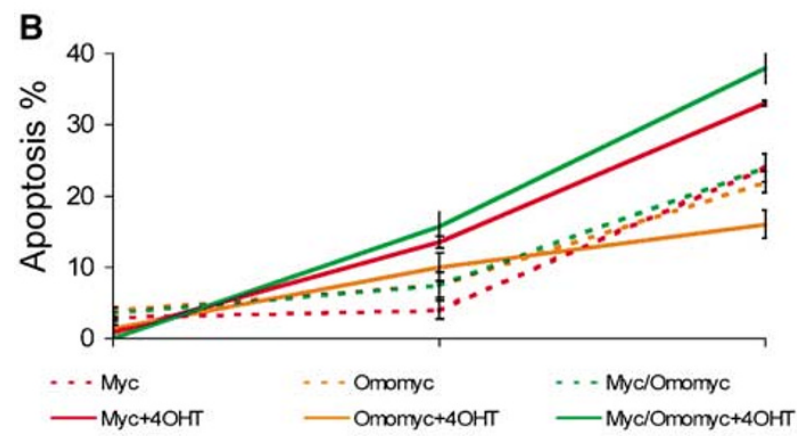

Figure 7 Omomyc potentiates Myc-induced apoptosis both in vitro and in vivo. (A) Omomyc potentiate Myc-dependent apoptosis of keratinocytes in vivo. Paraffin-embedded sections from Inv-c-MycER ${ }^{\text {TAM }}$, Inv-Omomyc single transgenic and Inv-c-MycER $R^{\text {TAM}} / O$ momyc double transgenic mice were stained for apoptosis by TUNEL. In skin of control Inv-c-MycER ${ }^{T A M}$ transgenic mice treated with ethanol (a) and in Inv-Omomyc single transgenics (b) practically no TUNEL-

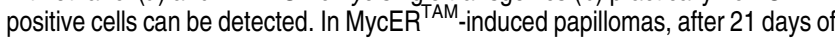
treatment with $4 \mathrm{OHT}$, a very small percentage of TUNEL-positive cells is detectable but restricted to the inner root sheath (c). In Inv-c-MycER $R^{\text {TAM } / O m o m y c}$ double transgenic mouse skin, extensive apoptotic staining is evident throughout the epidermis (d). (B) Omomyc potentiate Myc-dependent apoptosis of keratinocytes in vitro. Keratinocytes isolated from the same mice as in $(\mathbf{A})$ above were cultured in $1 \% \mathrm{FBS}$, either in the presence or absence of $4-\mathrm{OHT}$. Keratinocytes from all genetic backgrounds cultured in ethanol display little apoptosis, as do Omomyc-expressing keratinocytes cultured in presence of 4$\mathrm{OHT}$ (Omomyc + 4-OHT). In contrast, activation of MycER ${ }^{\text {TAM }}$ in Inv-cMycER ${ }^{T A M}$ keratinocytes results in significant apoptosis (Myc $+4-\mathrm{OHT}$ ) which is further exacerbated in inv-MycER ${ }^{\text {TAM }} /$ Omomyc double transgenic keratinocytes treated with 4-OHT (Myc/Omomyc + 4-OHT). hf, hair follicle; apo, apoptotic cell

The short half-life of apoptotic cells in intact skin tissue in vivo makes accurate quantitation of apoptosis by techniques like TUNEL very difficult. ${ }^{18}$ For this reason, we confirmed the potentiation of Myc-induced keratinocyte apoptosis by Omomyc in vitro, where apoptotic cells persist and can be more easily quantitated, and where the availability of exogenous survival factors can be precisely controlled. Keratinocytes from the various normal and transgenic mice were isolated and cultured in $1 \%$ serum, in either the presence or absence of 4-OHT to regulate MycER ${ }^{\text {TAM }}$ activity. Flow cytometric analysis was then used to quantitate apoptotic sub-G1 populations in each case. Even though Myc is unable to induce apoptosis in keratinocytes in intact epidermis in vivo, as previously reported, ${ }^{11}$ it retains the ability to do so in vitro when survival factors are depleted, as in low serum. Such 
Myc-induced apoptosis is evident as a subdiploid population in MycER ${ }^{\mathrm{TAM}}$ expressing keratinocytes in vitro following treament with 4-OHT. The extent of this Myc-dependent apoptosis was significantly enhanced in inv-c-MycER $R^{T A M}$ / Omomyc expressing keratinocytes treated with 4-OHT (Figure 4B). These results demonstrate that Omomyc does indeed promote c-Myc-dependent apoptosis in keratinocytes both in vitro and in vivo and, most significantly, in a tissue in which apoptosis is normally suppressed. ${ }^{11}$

\section{Omomyc restores the homeostatic balance between proliferation and apoptosis in skin, suppressing the oncogenic action of c-Myc}

Activated Myc is a potent inducer of keratinocyte proliferation. To assess the proliferative status of keratinocytes in skin of the various transgenic mice, we stained the tissues with antibodies to Ki67, which is expressed in all phases of the proliferating cell cycle. ${ }^{19}$ In normal skin, the proliferative compartment is typically confined to the basal layer. Likewise, suprabasal Ki67 staining was completely absent in the epidermis of untreated inv-c-MycER $R^{T A M}$ or inv-Omomyc single transgenic mice. In sharp contrast, however, Ki67 staining extended well into the hyperplasias and papillomas induced by sustained MycER ${ }^{\mathrm{TAM}}$ activation in Inv-c-MycERTAM mice (Figure 8). Interestingly, the epidermis of inv-c$M y c E R^{T A M} /$ Omomyc double transgenic mice treated with 4-OHT exhibited not only an increased number of suprabasal apoptotic cells but also a significant increase in Ki67-positive cells in the basal compartment.

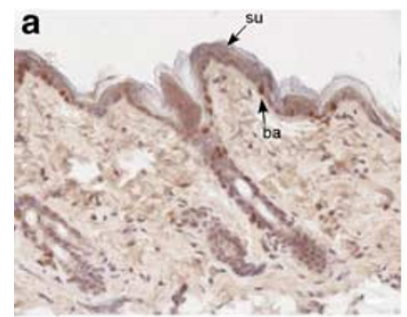

MycERTAM+EtOH

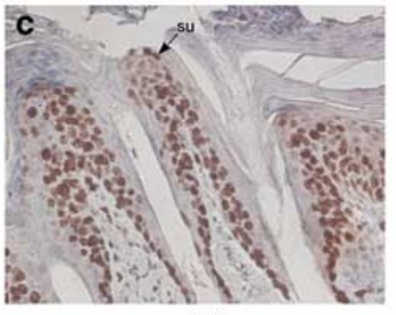

MycERTAM+OHT

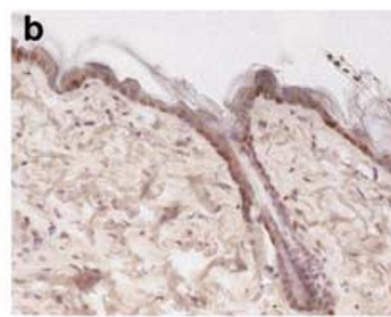

Omomyc+OHT

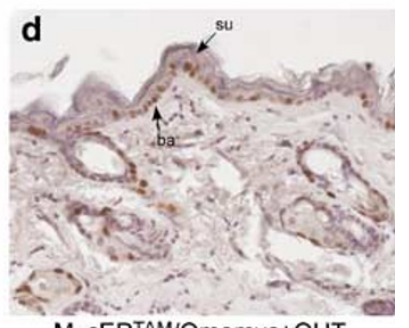

MycERTAM/Omomyc+OHT
Figure 8 Omomyc restores the balance between proliferation and apoptosis disrupted by Myc activation. Immunohistochemical staining of dorsal skin sections with Ki67 antibody. Proliferation is confined to basal keratinocytes in both ethanol-treated Inv-MycER ${ }^{\text {TAM }}$ mice (a) and 4-OHT-treated inv-Omomyc single transgenics (b). Hyperplastic skin (c) from inv-MycER ${ }^{T A M}$ mice treated for 21 days with daily 4-OHT exhibits Ki67-positive cells well out into the hyperplastic suprabasal layers. In contrast, proliferating cells in 21 day 4-OHT-treated inv$M y c E R^{T A M} /$ Omomyc double transgenic skin remains confined to the basal layer, although their number is increased relative to that in control mouse skin (d). su, suprabasal layer; ba, basal layer

\section{Discussion}

The obligate dual proliferative and apoptotic pathways elicited by Myc are thought to represent an intrinsic safeguard against neoplasia. However, the molecular basis underlying the apoptotic pathway that Myc implements is not well understood. Recently, we showed that the Myc bHLHZip mutant Omomyc can potentiate Myc-induced apoptosis even though it inhibits Myc-binding to the E-box. ${ }^{8}$ Given this peculiar property, we decided to investigate which putative Myc effector pathways might mediate such apoptotic activity. Our data indicate that Omomyc-induced apoptosis can annul protection conferred by overexpression of $\mathrm{Bcl}-\mathrm{x}_{\mathrm{L}}$, even though this is sufficient to block apoptosis induced by Myc alone. Thus, Omomyc can bypass or overwhelm the controls that trigger the mitochondrial apoptotic mechanism. Moreover, unlike apoptosis induced by Myc alone, which requires both ARF and p53, Omomyc-dependent apoptosis does not require $A R F$, although p53 remains necessary. This observation is particularly interesting from a therapeutic stance, since known Myc-dependent tumors such as Burkitt lymphomas typically harbor defects in the p14 ${ }^{\mathrm{ARF}}-\mathrm{MDM} 2-\mathrm{p} 53$ pathway as well as alterations in components of the basic apoptotic mitochondrial machinery such as loss of Bax or elevated expression of Bcl-2. ${ }^{20,21}$ Since Omomyc inhibits Myc binding to its E-box consensus DNA binding site, we surmise that its proapoptotic action is most likely related to Myc transrepression functions. ${ }^{8}$ However, we cannot exclude the possibility that Omomyc has additional properties. In any case, it will be important to identify the molecular targets of Myc-Omomyc heterodimers since they clearly play important roles in determining cell fates.

Significant evidence indicates that suppression of Mycinduced apoptosis is mandatory for the tumorigenic potential of Myc to become manifest (reviewed in Evan and Vousden ${ }^{22}$ ). Indeed, in some tissues, such as pancreatic $\beta$ cells, activation of Myc triggers rapid apoptotic involution of the entire tissue and suppression of Myc-induced apoptosis is sufficient to foster rapid cell expansion and malignant progression. ${ }^{23}$ In other tissues, such as skin epidermis, activation of Myc (and, by implication other oncoproteins) implements a transcriptional program for apoptosis but execution of cell death is then suppressed by endogenous survival factors. ${ }^{11}$ In all instances, however, restoration or reinforcement of Myc-induced apoptosis offers the potential of a powerful therapeutic strategy that would, moreover, be tumor specific since it relies on the presence of the underlying tumorigenic Myc lesion. We have now validated this therapeutic concept by demonstrating that coexpression of Omomyc together with Myc in suprabasal skin, a tissue in which Myc-induced apoptosis is innately suppressed, profoundly tips the balance between proliferation and apoptosis towards cell death, thereby thwarting the oncogenic action of Myc in this tissue. This remarkable attribute of Omomyc remains completely dependent on the presence of a deregulated Myc, however, since constitutive expression of Omomyc alone in normal skin epidermis has no detectable effect. The end result is restoration of an apparently normal balance between cell gain and loss, presumably orchestrated by the basal cells which are able to maintain the requisite 
proliferative rate necessary to compensate for loss of the outer epidermal layers. The dramatic reprogramming induced by Omomyc is sufficient to prevent both papilloma development and all other phenotypic changes associated with precancerous epithelial lesions caused by Myc, including dysplasia and angiogenesis. This striking result underscores the extraordinary capacity of skin to maintain its architecture in spite of a highly variable degree of cellular turnover. It may be that such increased cell turnover is not compatible with the maintenance of tissue integrity and homeostasis in all other tissues. However, our data indicate that any proapoptotic effect of Omomyc will be limited only to cells harboring activated Myc, so any effect of Omomyc is likely to be restricted only to tumor cells. Such studies clearly demonstrate the potential of using the innate tumor-suppressive attributes of existing oncogenic effector molecules, such as Myc, to redirect or reprogram their functions for therapeutic purposes.

\section{Materials and Methods}

\section{Expression vectors and cell lines}

All cell lines were grown in Dulbecco's modified Eagle medium (DMEM) supplemented with $10 \%$ FCS. Normal Rat- 1 or Rat-1/Bcl- $x_{L}$, and normal,

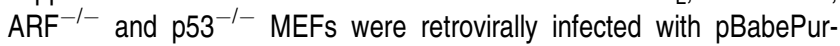
oMycER $^{\text {TAM }}$, then selected with $2.5 \mu \mathrm{g} / \mathrm{ml}$ puromycin. The MycER ${ }^{\text {TAM }}$ construct was activated with $100 \mathrm{nM}$ of 4-hydroxytamoxifen (4-OHT), $24 \mathrm{~h}$ before serum deprivation. Omomyc DNA was cloned into the $p B M N$-IRESGFP retroviral vector.

\section{Flow cytometric analysis of apoptosis and cell sorting}

Flow cytometric analysis and sorting of pBMNOmomyc-IRES-GFP cells were performed according to published protocols. ${ }^{13}$

\section{Transgene construction}

Omomyc DNA, carrying an ATG start codon, ${ }^{7,8}$ was amplified from the pBPOmomyc construct by PCR primed using oligonucleotides containing the Notl restriction site. The resulting Omomyc sequence was fused to the Involucrin promoter, into the Notl site of the Involucrin expression cassette. ${ }^{11}$ The inv-Omomyc transgene was excised from its parent plasmid as a Sall fragment, isolated and then purified for microinjection.

\section{Generation of transgenic mice}

inv-Omomyc transgene DNA was injected into the male pronucleus of day 1-fertilized (CBA X C57BL/6) $F_{1}$ embryos. Injected embryos were transferred into day 1-plugged pseudopregnant foster mice, and litters were screened for the presence of the transgene by PCR using primers matching the Involucrin promoter and Omomyc sequences.

\section{Antibodies}

Rabbit polyclonal anti-Omomyc antibody was raised against a synthetic peptide comprising amino acids 66-78 of the Omomyc polypeptide and including the signature 4 amino-acid substitutions that characterize Omomyc. The specificity of this antibody (Omomyc1) was confirmed by
ELISA on specific versus several irrelevant peptides. The antibody was unable to recognize Myc in Western blots of cell extracts derived from Myc overexpressing cells. Other primary antibodies used were: ER-P2 (raised against the carboxy terminal 20 amino acids of the murine estrogen receptor), mouse keratins 1 (K1) and 14 (K14) (Covance), and Ki67 (Immunotech).

\section{Histology and immunohistochemical staining of mouse skin}

Skin samples were obtained from transgenic or wild-type mice matched according to age and body site, and tissues were fixed overnight in neutralbuffered formalin, embedded in paraffin, sectioned $(5 \mu \mathrm{m})$, and stained with hematoxylin and eosin. For antigen retrieval, sections were microwaved in $0.01 \mathrm{M}$ citrate buffer $(\mathrm{pH} 6.0)$. For all antibody staining, an $A B C$ Vectastain Elite kit (Vector Laboratories) was used according to the manufacturer's instructions. Sections were counterstained in hematoxylin for $1 \mathrm{~min}$. Immunological specificity of staining with antiOmomyc was confirmed by preincubating the antibody with its cognate immunogenic peptide $(100 \mathrm{nM})$ for $30 \mathrm{~min}$ at RT prior to use, which abrogated all measurable binding to sections of Omomyc-expressing tissue. Apoptosis was assessed in tissue sections by TUNEL staining using the ApopTag kit (Intergen) according to the manufacturer's instructions, after which the sections were counterstained with methylene blue.

\section{Isolation and culture of mouse keratinocytes}

Mouse keratinocytes were isolated as described. ${ }^{24}$ Keratinocytes were cultured in EMEM containing $0.05 \mathrm{mM} \mathrm{CaCl}_{2}$ and supplemented with $10 \%$ or $1 \% \mathrm{FBS}, 0.5 \mu \mathrm{g} / \mathrm{ml}$ hydrocortisone, $5 \mu \mathrm{g} / \mathrm{ml}$ insulin, and $0.1 \mu \mathrm{g} / \mathrm{ml}$ gentamycin.

\section{Administration of 4-hydroxytamoxifen (4-OHT)}

MycER $^{\text {TAM }}$ was activated in the skin of adult transgenic mice by topical application of $1 \mathrm{mg} \mathrm{4-OHT} \mathrm{(Sigma)} \mathrm{dissolved} \mathrm{in} \mathrm{ethanol}(0.2 \mathrm{ml})$ to a shaved area of dorsal skin. Control mice were treated with ethanol alone.

\section{Acknowledgements}

We are grateful to Lamorna Brown Swigart, Ignacio Flores and Maria Christophorou for their technical assistance, to Daniel J Murphy, Julia E Prescott and the other members of the Evan Laboratory for helpful discussions. SN was supported by grants from AIRC and from MIUR-FIRB project.

\section{References}

1. Eisenman RN (2001) Deconstructing myc. Genes Dev. 15: 2023-2030

2. Lutz W, Leon $\mathrm{J}$ and Eilers $\mathrm{M}$ (2002) Contributions of Myc to tumorigenesis. Biochim. Biophys. Acta. 1602: 61-71

3. Mateyak MK, Obaya AJ, Adachi S and Sedivy JM (1997) Phenotypes of c-Mycdeficient rat fibroblasts isolated by targeted homologous recombination. Cell Growth Differ. 8: 1039-1048

4. Trumpp A, Refaeli Y, Oskarsson T, Gasser S, Murphy M, Martin GR and Bishop JM (2001) c-Myc regulates mammalian body size by controlling cell number but not cell size. Nature 414: 768-773 
5. Evan $G$ and Littlewood T (1998) A matter of life and cell death. Science 281 1317-1322

6. Askew DS, Ashmun RA, Simmons BC and Cleveland JL (1991) Constitutive cmyc expression in an IL-3-dependent myeloid cell line suppresses cell cycle arrest and accelerates apoptosis. Oncogene 6: 1915-1922

7. Soucek L, Helmer-Citterich M, Sacco A, Jucker R, Cesareni $G$ and Nasi S (1998) Design and properties of a Myc derivative that efficiently homodimerizes. Oncogene 17: 2463-2472

8. Soucek L, Jucker R, Panacchia L, Ricordy R, Tato F and Nasi S (2002) Omomyc, a potential Myc dominant negative, enhances Myc-induced apoptosis. Cancer Res. 62: 3507-3510

9. Juin P, Hueber AO, Littlewood T and Evan G (1999) c-Myc-induced sensitization to apoptosis is mediated through cytochrome $c$ release. Genes Dev. 13: 1367-1381

10. Sherr CJ (1998) Tumor surveillance via the ARF-p53 pathway. Genes Dev. 12 2984-2991

11. Pelengaris S, Littlewood T, Khan M, Elia G and Evan G (1999) Reversible activation of c-Myc in skin: induction of a complex neoplastic phenotype by a single oncogenic lesion. Mol. Cell. 3: 565-577

12. Kluck RM, Bossy-Wetzel E, Green DR and Newmeyer DD (1997) The release of cytochrome $c$ from mitochondria: a primary site for bcl-2 regulation of apoptosis. Science 275: 1132-1136

13. Klefstrom J, Verschuren EW and Evan G (2002) c-Myc augments the apoptotic activity of cytosolic death receptor signaling proteins by engaging the mitochondrial apoptotic pathway. J. Biol. Chem. 277: 43224-43232

14. Zindy F, Eischen CM, Randle DH, Kamijo T, Cleveland JL, Sherr CJ and Roussel MF (1998) Myc signaling via the ARF tumor suppressor regulates p53-dependent apoptosis and immortalization. Genes Dev. 12: 2424-2433
15. Carroll JM, Albers KM, Garlick JA, Harrington R and Taichman LB (1993) Tissue- and stratum-specific expression of the human involucrin promoter in transgenic mice. Proc. Natl. Acad. Sci. USA 90: 10270-10274

16. Johnson LD, Idler WW, Zhou XM, Roop DR and Steinert PM (1985) Structure of a gene for the human epidermal $67-\mathrm{kDa}$ keratin. Proc. Natl. Acad. Sci. USA 82: $1896-1900$

17. Nelson WG and Sun TT (1983) The 50- and 58-kdalton keratin classes as molecular markers for stratified squamous epithelia: cell culture studies. J. Cell Biol. 97: 244-251

18. Gandarillas A, Goldsmith LA, Gschmeissner S, Leigh IM and Watt FM (1999) Evidence that apoptosis and terminal differentiation of epidermal keratinocytes are distinct processes. Exp. Dermatol. 8: 71-79

19. Schluter C, Duchrow M, Wohlenberg C, Becker MH, Key G, Flad HD and Gerdes J (1993) The cell proliferation-associated antigen of antibody Ki-67: a very large, ubiquitous nuclear protein with numerous repeated elements, representing a new kind of cell cycle-maintaining proteins. J. Cell Biol. 123: 513-522

20. Lindstrom M and Wiman K (2002) Role of genetic and epigenetic changes in Burkitt lymphoma. Semin. Cancer Biol. 12: 381

21. Cory S, Vaux DL, Strasser A, Harris AW and Adams JM (1999) Insights from $\mathrm{Bcl}-2$ and Myc: malignancy involves abrogation of apoptosis as well as sustained proliferation. Cancer Res. 59: 1685s-1692s

22. Evan GI and Vousden KH (2001) Proliferation, cell cycle and apoptosis in cancer. Nature 411: 342-348

23. Pelengaris S, Khan M and Evan Gl (2002) Suppression of Myc-induced apoptosis in beta cells exposes multiple oncogenic properties of Myc and triggers carcinogenic progression. Cell 109: 321-334

24. Leigh I and Watt F (1994) Keratinocyte Methods. (Cambridge: University Press) pp. 25-31 